\section{AB001. Understanding transcriptional connections of chronic cutaneous lupus erythematosus between humans and animal models}

\author{
Frane Banovic ${ }^{1}$, Amanda Blubaugh ${ }^{1}$, Tara Denley ${ }^{1}$, \\ Robert M. Gogal ${ }^{2}$
}

${ }^{1}$ Department of Small Animal Medicine and Surgery, University of Georgia College of Veterinary Medicine, Athens, GA, USA; ${ }^{2}$ Department of Biosciences and Diagnostic Imaging, University of Georgia College of Veterinary Medicine, Athens, GA, USA

Correspondence to: Frane Banovic. College of Veterinary Medicine, University of Georgia, 2200 College Station Road, 30605 Athens, GA, USA. Email: fbanovic@uga.edu.

Abstract: Chronic cutaneous lupus erythematosus (CCLE) exhibits severe inflammatory processes that lead to decreased quality of life (QoL) and potential skin scarring with disfiguration; treatment of CCLE has a high unmet need for effective and safe therapeutics. To determine biological pathways and transcriptional networks unique to CCLE, we performed meta-analysis of published humans CCLE transcriptome studies and comparative correlation with spontaneous canine and murine MRL/lpr model CCLE skin lesions. Utilizing published microarray data of three human CCLE studies, we determined a comprehensive consensus CCLE shared gene list of 245 genes differentially expressed genes (DEGs) (>2-fold enhanced, $\mathrm{P}<0.05)$. The Th1 and interferon-related genes (STAT1, OASL, MX1, IFN $\gamma$, GZMB, ISG15) as well as T-cell trafficking chemokines, CXCL9, CXCL10 and
CCL11, were among the strongest upregulated genes. Top enriched process networks by MetaCore overlap analysis of human CCLE studies revealed upregulation of interferon and IFNy signaling, innate immune response to RNA viral infection, NK-cell cytotoxicity and JAK-STAT pathway. Comparative analysis between canine and mouse CCLE DEGs with the human CCLE meta-analysis derived DEG list found that canine and murine CCLE lesions contained $57 \%(139 / 245)$ and 24\% (59/245) shared genes, receptively. Spearman correlation coefficients of canine CCLE DEGs with three human CCLE studies showed significant moderate to strong positive correlation $(\mathrm{r}=0.52-0.67)$. The shared canine CCLE DEGs within the human CCLE lesional pathology signature reflected strongly activated pathway maps of IFN $\alpha / \beta$ signaling via JAK-STAT, antiviral actions of IFN and IFN $\gamma$ signaling. In conclusion, canine spontaneous CCLE model appears the best to replicate human CCLE immune signatures.

Keywords: Cutaneous lupus; human; mouse; canine

doi: 10.21037/atm.2021.AB001

Open Access Statement: This is an Open Access article distributed in accordance with the Creative Commons Attribution-NonCommercial-NoDerivs 4.0 International License (CC BY-NC-ND 4.0), which permits the noncommercial replication and distribution of the article with the strict proviso that no changes or edits are made and the original work is properly cited (including links to both the formal publication through the relevant DOI and the license). See: https://creativecommons.org/licenses/by-nc-nd/4.0/.

Cite this abstract as: Banovic F, Blubaugh A, Denley T, Gogal RM. Understanding transcriptional connections of chronic cutaneous lupus erythematosus between humans and animal models. Ann Transl Med 2021;9(5):AB001. doi: 10.21037/atm.2021.AB001 